\title{
De la révision du Dictionnaire de Samuel Galley*
}

Pierre Ondo-Mebiame, Département des Sciences du Langage et Groupe de Recherche en Langues et Cultures Orales (GRELACO), Université Omar Bongo, Libreville, République Gabonaise (pierreondo@yahoo.fr)

Résumé: Le Dictionnaire fang-français et français-fang de Samuel Galley (1964) constitue une contribution importante dans l'histoire lexicographique du parler et, par sa nature biscopale, un intérêt pour les locuteurs du fang. Au demeurant, il présente un nombre important de lacunes qui méritent d'être corrigées. En l'occurrence, et entre autres, le lexicographe n'a pas mentionné les critères selon lesquels les items lexicaux ont été lemmatisés dans le dictionnaire. Par conséquent, on est forcé de deviner les sources du dictionnaire. Le traitement des mots composés et des homonymes doit également être amélioré. Ce sont ces améliorations que nous esquissons dans la présente proposition.

Mots clés: DICTIONNAIRE, LEXICOGRAPHIE, SOURCE, PRÉ-TEXTES, NOMENCLATURE, POSTTEXTES, SIGNE, LEMMES, BISCOPAL, MACROSTRUCTURE, MICROSTRUCTURE, ENTÊTE, PARAPHRASE, MOT COMPOSÉ, HOMONYMES

Abstract: About the Revision of Samuel Galley's Dictionary. Samuel Galley's Dictionnaire fang-français et français-fang (1964) forms an important contribution towards the lexicographic history of the language and, because of its biscopal nature, is of interest to speakers of Fang. However, it shows a number of important shortcomings, which should be corrected. Among others, the lexicographer does not mention the criteria according to which lexical items have been lemmatized in the dictionary. Therefore one is compelled to guess the sources of the dictionary. The treatment of compound words and homonyms needs also to be improved. These improvements are outlined in this contribution.

Keywords: DICTIONARY, LEXICOGRAPHY, SOURCE, FRONT MATTER TEXTS, CENTRAL LIST, BACK MATTER TEXTS, SIGN, LEMMATA, BISCOPAL, MACROSTRUCTURE, MICROSTRUCTURE, HEADWORDS, PARAPHRASE, COMPOUND WORD, HOMONYMS

\section{Présentation}

\subsection{Généralités}

Selon Mihindou (2001: 12) le texte de Galley intitulé Dictionnaire fang-français et français-fang est l'un des dictionnaires bilingues produit en fang du Gabon. Il

* Cet article fait suite à la contribution de Mavoungou (2001) intitulée "Macro- and Microstructural Issues in Mazuna Lexicography" en Lexikos 11: 122-138. 
s'agit d'une œuvre produite à titre posthume, grâce au concours conjugué de l'Association Française des Amis d'Albert Schweitzer, de la veuve Galley, et du professeur Henry A.C. Samuel Galley, qui, pour mémoire, était un Missionnaire de l'Église protestante et travailla en pays fang pendant près de quarante ans.

Toujours selon Mihindou (2001: 12), le texte de Galley peut être divisé en trois parties:

(1) les pré-textes;

(2) la nomenclature; et

(3) les posttextes.

Les pré-textes comprennent:

(a) le titre de l'ouvrage, le nom de l'auteur, l'initiateur de la publication, l'éditeur et le lieu de l'édition;

(b) la date de la fin d'impression, le nom et le lieu de l'imprimerie; et

(c) la préface et l'avant-propos.

La nomenclature ou dictionnaire proprement dit observe l'ordre alphabétique, et comprend deux parties:

(a) la présentation des lemmes en utilisant le fang comme idiome source; et

(b) la présentation des lemmes en utilisant le français comme langue source.

Les posttextes enfin, sont un exposé sur la grammaire du fang rendu en sept chapitres, à savoir:

(a) l'orthographe et la phonétique;

(b) les classificateurs des noms;

(c) les classes des noms;

(d) les adjectifs;

(e) les pronoms;

(f) les verbes; et

(g) les mots invariables.

En dehors de ses activités lexicographiques et évangéliques, le pasteur Samuel a également entrepris la production des ouvrages didactiques. Son outil didactique le plus connu est le livre Nteni Osu Nteni Fañwe ${ }^{1}$ publié en 1889 sous l'égide de la station protestante de Talagouga. 


\subsection{Avantages}

Le dictionnaire de Galley est prévu pour satisfaire à la fois les besoins des locuteurs du fang et du français en raison de sa nature biscopale (le terme est emprunté à Hausmann et Werner 1991: 2740) ou bi-directionnelle. Sa nomenclature présente une macrostructure dont les lemmes (ou unités du dictionnaire) sont rangés dans l'ordre alphabétique, alors que la microstructure fournit un nombre important de données, dont:

- lindication des parties du discours;

- le numéro de la classe de nom;

- les traductions,

- la paraphrase du sens; et

- les renvois.

Comparons, pour illustrer, l'article du lemme AKÔL:

AKÔL (h) n.4, pl. mekôl. Arbre dont les feuilles sont rigueuses et peuvent remplacer la toile émeri ou le papier de verre. Dans les écoles, les ardoises sont souvent remplacées par de petites planchettes en ékukh; les élèves écrivent dessus avec le crayon à mine de plomb et effacent bruyamment avec les feuilles d'akôl. Akôl ntañan, véritable papier de verre ou toile émeri.

Exemple textuel 1: Article AKÔL (extrait du Galley² 1964: 30)

Une autre caractéristique du dictionnaire est la structure d'accès. En effet, l'utilisation des marqueurs typographiques tels que la présentation des lemmes en gras et en lettres majuscules, ainsi que les mots-guides ou entêtes qui forment la structure externe d'accès rapide (Hausmann et Wiegand 1989: 329) facilitent aux utilisateurs, la quête du mot qu'ils recherchent.

$\mathrm{Au}$-delà de ces avantages, notre dessein est d'inviter à une révision de l'importante proposition de Galley, en indiquant les aspects à améliorer. Nos suggestions porteront sur les plans macrostructurels et microstructurels.

\section{La macrostructure du dictionnaire de Galley}

\subsection{La lemmatisation des items}

La macrostructure du Dictionnaire fang-français et français-fang contient \pm 13925 articles présentés dans l'ordre alphabétique strict. C'est un recueil du parler fang tel qu'il est utilisé par la communauté de Talagouga ${ }^{3}$.

En amont du dictionnaire, le lexicographe a pris en compte les mots utilisés dans le discours quotidien, les noms géographiques, les noms de plantes et d'animaux, les noms propres et les expressions idiomatiques en tant que 
partie intégrante de ses éléments macrostructuraux. Le texte est donc une collection représentative des items lexicaux du fang en usage à Talagouga. Le lexicographe aura voulu par là, que son dictionnaire soit, selon l'expression de McArthur (1986) un "véritable réservoir de connaissances".

Au demeurant, ce dernier (en l'occurence l'éditeur) n'a pas mentionné les critères selon lesquels les items lexicaux ont été lemmatisés dans le dictionnaire. Ce genre d'informations est habituellement présenté dans le guide, à l'endroit des usagers, qui constitue les pré-textes du dictionnaire. L'on observe que la préface du dictionnaire fait simplement état des raisons pour lesquelles l'Association Française des Amis d'Albert Schweitzer a décidé de publier le travail du pasteur Genevois. La section introductive donne également un aperçu des activités de missionnaires, des lexicographes et des pédagogues de Galley. Rien n'est cependant mentionné au sujet du choix des lemmes.

L'on est donc, fort de cela, en droit de s'interroger sur:

- Les sources primaires et secondaires du dictionnaire;

- Les utilisateurs potentiels du dictionnaire; et

— Les besoins et les aptitudes des utilisateurs-cibles du dictionnaire.

Comme le lecteur avisé pourrait s'en apercevoir, ces interrogations ne sont pas prises en charge par les pré-textes du dictionnaire; par conséquent, l'on est obligé de deviner les sources du dictionnaire.

\subsection{Les sources primaires et secondaires du dictionnaire}

Au sujet des sources primaires ${ }^{4}$, le guide du dictionnaire nous laisse comprendre que le travail est basé sur la traduction de la Bible en fang que Galley a entreprise pendant 40 ans pour le compte de la communauté fang de Talagouga. Une mention est également faite à l'aide de l'interprète de Galley: Obam Mendôme.

Les sources secondaire ${ }^{5} \mathrm{du}$ dictionnaire ne sont pas explicitement discutées dans le guide aux usagers. La préface mentionne cependant l'existence d'un travail lexicographique antérieur avec des éléments macrostructuraux restreints. Il s'agit très probablement du Lexique fãn-français compilé par Martrou (1924).

On trouve une mention des sources secondaires en examinant minutieusement la nomenclature, et spécifiquement l'article MVEP. L'examen de ce lemme donne:

MVEP (h) n.3, pl. bemvep Rongeur, appelé en anglais canerat, rat de canne à sucre ., Largeau dit le même mot.

Exemple textuel 2: Article MVEP (extrait du Galley 1964: 212) 
Le traitement de ce lemme-là produit donc la preuve que Galley a utilisé l'Encyclopédie pahouine de Largeau (1901) comme source secondaire, au cours de l'élaboration de son dictionnaire. Pour la gouverne, il est bon de savoir que l'ouvrage de Largeau porte sur les variétés fang-atsi et fang-make. On pourrait même aller plus loin et penser que Galley ne s'est pas uniquement limité à l'exploitation de l'encyclopédie de Largeau, mais a employé tous les ouvrages lexicographiques antérieurs portant sur le fang ${ }^{6}$. Ce soupçon est plus évident dans la section français-fang. Il semble en effet que les dictionnaires monolingues ou bilingues du français qui ont été publiés avant le texte de Galley aient constitué des sources secondaires de son ouvrage.

\subsection{Les utilisateurs potentiels du dictionnaire et leurs besoins et aptitudes}

La section introductive mentionne le fait que le dictionnaire ne vise pas un public sophistiqué en ce que l'auteur n'a pas proposé la transcription phonétique de ses unités de traitement. Mais il n'en demeure pas moins, à l'usage, que l'ouvrage soit également conçu pour satisfaire les besoins des étudiants, des chercheurs, et en même temps, des personnes qui souhaitent apprendre la langue.

André-Charles Henry, le préfacier de l'œuvre de Galley, déclare à ce propos que l'ouvrage de Galley n'est pas un livre pour les personnes savantes. En effet, la langue, ou mieux, le niveau de langue utilisé dans le dictionnaire est tout sauf hermétique. Les paraphrases du sens utilisées sont simples et concises.

Au demeurant, l'inclusion de données telles que le numéro de classe, la formation du mot, les marqueurs structuraux, l'étymologie, les synonymes, les homonymes et même les paraphrases du sens, présupposent une sérieuse connaissance linguistique de la part de l'utilisateur-cible, malgré l'insertion d'une mini-grammaire en posttexte. Cela fait donc penser que les lecteurs-cibles du dictionnaire pourraient d'être des chercheurs et des étudiants.

\section{La microstructure du dictionnaire de Galley}

Nous allons traiter de la microstructure du dictionnaire de Galley en exposant les avantages et les inconvénients.

\subsection{Avantages}

(1) La microstructure du dictionnaire de Galley, et cela est de notoriété (Mihindou 2001) présente beaucoup d'informations. On relève en l'occurrence:

- les parties du discours;

- les numéros de classes des noms;

- les traductions; 
- les explications de sens; et

- les renvois.

(2) En outre, et cela n'a pas été suffisamment dit jusqu'ici, chaque signe-lemme est immédiatement et systématiquement suivi par une indication de tonalité, une entrée d'une grande importance pour l'utilisateur. Très souvent, les lexicographes produisant des dictionnaires dans des langues africaines ont toujours péché en ignorant cette composante de base de l'analyse phonologique de la langue.

Galley distingue trois niveaux de tonalité dans son dictionnaire:

(a) la tonalité élevée (notée (h));

(b) la tonalité basse (notée (b)); et

(c) la tonalité moyenne (notée $(\mathrm{m})$ ).

(3) On observe qu'en traitant de la signification, l'auteur fait l'effort de faire apparaître des données ayant trait à des distinctions dans la signification. Il expose donc ce qui se rapporte aux valeurs polysémiques d'un item lexical (cf. Bureau du WAT 1999). Considérons pour cela le lemme suivant:

AKÔNA (bm) n.4, pl. mekôna (vbs kon h et wôm h). Amas, agglomération, quelque
chose qui forme un bloc. Akôna ndôa, brasier out rouge. Akôna ñkur, amas de
nuages. Akôna mekî, caillot de sang. Akôna mezim, bouillonnement d'eau dans les
rapides. Akôna kôn, myaña, lingot d'or, d'argent. Akôna éki, lingot de fer parfois
donné parmi d'autres choses comme dot (syn.: awur ékî). Akôna ékî signifie aussi
métal quelconque qui a été fondu. Voir ñkôna.

Exemple textuel 3: Article AKÔNA (extrait du Galley 1964: 30)

Les syntagmes Akôna ndôa, Akôna ñkur, Akôna mekî, Akôna mezim, Akôna kôñ, myaña et Akôna éki se rapportent aux expansions polysémiques de la signification du lemme AKÔNA ('Amas, agglomération, quelque chose qui forme un bloc').

(4) Une autre caractéristique du dictionnaire se situe au niveau du traitement des signes-lemmes représentant les items lexicaux polysémiques. Parcourons le traitement du lemme ABEÑY.

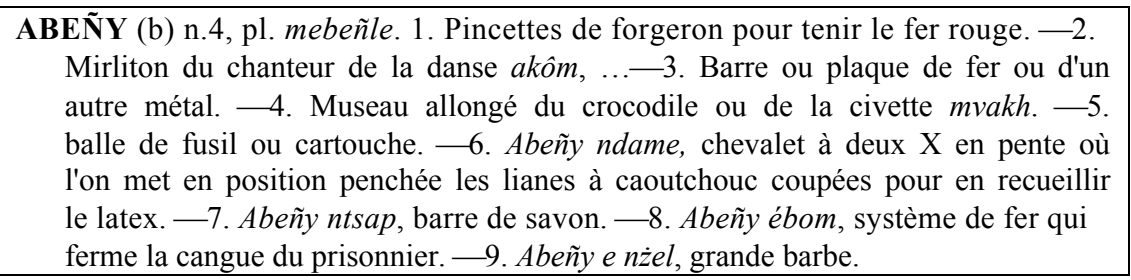

Exemple textuel 4: Article ABEÑY (extrait du Galley 1964: 16) 
Dans l'exemple 4, le lexicographe fournit aux utilisateurs des données présentées dans deux sections de l'article:

(a) le "commentaire sur la forme" (CF); et

(b) le "commentaire sur le sens" (CS) (cf. Hausmann et Wiegand 1989: 353).

Les entrées telles que le signe-lemme (ABEÑY), la classe de tonalité (b), le numéro de classe (n.4) et l'indication du pluriel (mebeñle) qui apparaissent dans la structure gauche du noyau de l'article du dictionnaire, font partie du commentaire sur la forme ou CF; le reste des entrées dans la structure droite du noyau de l'article appartient au commentaire sur le sens CS (cf. Hausmann et Wiegand 1989: 353-354).

L'utilisateur peut considérer le lemme ABEÑY comme représentant un item lexical polysémique. Chaque sens est introduit par un indicateur ou marqueur structurel $1,2 \ldots 9$ qui fait partie de la structure interne d'accès rapide du dictionnaire (cf. Hausmann et Wiegand 1989: 354-356).

(5) Une autre caractéristique du dictionnaire porte sur le traitement des termes de spécialité et des concepts culturels. Comparez le traitement de l'article du lemme AKUA:

AKUA (b) n.4, pl. mekua. Endroit où on fait fondre le minerai de fer. On fait un grand trou dans la terre, on y met le minerai et beaucoup de charbon de bois. Tous ceux qui ont des soufflets de forge indigènes les installent autour du trou. On allume le brasier, on souffle sans arrêt jusqu'à ce que la fusion s'opère. On se relaie, et les femmes apportent des vivres aux souffleurs.

Exemple textuel 5: Article AKUA (extrait du Galley 1964: 31)

Le traitement lexicographique commence avec une paraphrase de la signification du lemme AKUA. Ensuite, une explication détaillée est donnée à propos de l'extraction du minerai de fer, ses acteurs, les instruments et techniques utilisés pour la circonstance. Ces explications, qui portent principalement sur les caractéristiques extra-linguistiques, ne font évidemment pas partie de la paraphrase de la signification.

(6) Il est important d'observer comment le lexicographe traite les noms de plantes et ceux des animaux. Considérons les exemples suivants:

TôM (b) n.3, pl. metôm. Grand arbre parasol. Nom commercial d'après Meniaud: Tsoumbou. (Nom scientifique: Parkia Klainei Pierre). Couleur du bois: gris jaune. Arbre magnifique à voir. Tôlba tôm (vb tole $\mathrm{h}$ ), se faire verser goutte à goutte une infusion de bois de tôm dans l'œil, comme épreuve pour savoir si l'adultère a été commis (ordalie).

Exemple textuel 6: Article TÔM (extrait du Galley 1964: 344) 
MVON (b) n.3, pl. bemvon. Gros singe à long poils noirs (fourrure), colobe (Colobus Satanas).

Exemple textuel 7: Article MVON (extrait du Galley 1964: 214)

L'exemple 6 contient le nom scientifique, une description et les utilisations de l'arbre et l'exemple 7 le nom scientifique et une description du singe.

Le traitement des termes de spécialité a été fait d'une manière tout à fait satisfaisante en ce qui concerne le contexte culturel parce que l'utilisateur a accès aux données lexicographiques concernant la faune et la flore de l'habitat naturel des populations Fang. Excepté quelques exemples (qu'il n'est pas nécessaire de mentionner ici), l'utilisateur du dictionnaire a toujours accès à des entrées telles que le nom scientifique (de la plante ou de l'animal concerné), les utilisations de la plante ainsi que les sources consultées.

Par ailleurs, les feuilles, les racines, les écorces, les fruits, les graines et les ingrédients d'animaux sont énumérés avec leurs utilisations dans la médecine traditionnelle. Toutes ces données concernant le contexte culturel des lemmes traités sont importantes pour l'utilisateur qui est susceptible de trouver des plantes et des ingrédients d'animaux en vente sur les étalages des marchés.

Dans la section français-fang du texte, les éléments macrostructuraux appartiennent très probablement au vocabulaire quotidien du français. La microstructure fournit habituellement à l'utilisateur-cible les données suivantes: la traduction en fang, parfois précédée par un synonyme ou des synonymes du lemme en français, et des exemples (chaque exemple en français est immédiatement suivi de son correspondant en fang). Les articles varient en longueur. Une illustration extrême de ce point, apparaît dans le traitement de l'article du lemme ARBRE (presque trois colonnes):

ARBRE bois, éli (h). Boîte en bois, évora éli (bh) ..

Exemple textuel 8: Article ARBRE (extrait du Galley 1964: 419-420)

La longueur de cet article résulte de l'énumération d'environ 200 essences d'arbres de la forêt gabonaise ainsi que leurs utilisations et descriptions. En outre, le nom commercial de l'arbre apparaît avec son nom local. Le lexicographe a essayé de traiter les items lexicaux de spécialité équitablement en ce qui concerne les deux sections du dictionnaire.

\subsection{Inconvénients}

(1) Ce que l'on observe, c'est que l'indication de tonalité est habituellement suivie d'une paraphrase du sens du lemme dans la langue-cible (le français). Ceci amène de notre point de vue, une discrimination car, une paraphrase du sens dans la langue-source (le fang) aurait donné aux locuteurs fang plus d'aptitudes dans la maîtrise du parler. Il semble donc que le dictionnaire est plus 
susceptible de satisfaire les besoins des locuteurs du français que ceux du fang. Comparons pour cela l'article du lemme MVIA:

MVIA (h) n.2, pl. mimvia (vb via h). Femme acquise avec la dot de notre fille. En d'autre termes, femme achetée par le beau-père avec la dot reçu de son gendre. Et sa fille dont la dot a été prise pour cela, c'est sa mbala (voir ce mot).

Exemple textuel 9: Article MVIA (extrait du Galley 1964: 213)

La paraphrase de sens ci-dessus, n'indique réellement à l'utilisateur que ce que signifie le lemme MVIA, et cette information n'est nullement utile au locuteurnatif, car il en dispose déjà a priori. Ce qu'il rechercherait, c'est l'amélioration de ses aptitudes.

(2) Dans l'article d'un signe-lemme représentant un item lexical polysémique comme pour l'exemple 4 ABEÑY, les différentes distinctions dans la signification ne devraient pas être présentées sur une base aléatoire, mais selon des critères fixes, qui devraient également être expliqués dans un des pré-textes. Or pour le lemme ABEÑY, on devine plutôt les principes qui gouvernent les différentes distinctions.

Il semble en effet que le lexicographe a distingué entre les significations primaires et secondaires. Ou plus probablement, l'arrangement du lemme ABEÑY est fait selon le principe des distinctions conjonctives et disjonctives dans la signification. Le sens 1 du signe-lemme en l'occurrence semble être la valeur polysémique littérale de l'item lexical; il est donc placé en premier dans l'article. Les sens 2, 3, 4, 5, 6, 7, 8 et 9 semblent être des extensions métaphoriques du premier sens. En outre, les sens 2, 3, 5, 6 et 8 ont des significations plus ou moins liées, parce qu'ils ont tous une relation avec l'item lexical renvoyant au fer ou à la métallurgie. Ainsi, ils auraient dû être arrangés par le lexicographe dans l'ordre mentionné ci-dessus.

Les sens 7 et 9 , dans lesquels la signification figurative ne se réfère pas directement au premier sous-commentaire sur le sens, pourraient avoir été ordonnés plus loin dans la liste.

Le lexicographe aurait également pu utiliser un autre principe d'arrangement, à savoir: le principe de l'arrangement empirique. Dans le principe de l'arrangement empirique, les distinctions dans la signification sont déterminées sur la base du calcul de fréquence (cf. Bureau du WAT 1999: 17-20).

(3) L'article du lemme ABEÑY met en relief quelques aspects de la présentation des mots composés. Abeñy ndame, Abeñy ntsap, Abeñy ébom et Abeñy e nżel sont des composés avec la structure: nom + (connectif) + nom. Tous ces mots composés sont orthographiés sans trait d'union.

À l'inverse, les lemmes MÔR-A-MVAM, MVAKH-FÎN et AKAM-NYAR sont présentés avec des traits d'union. Ceci est très déroutant puisque les lemmes en question sont des mots composés avec la structure, nom + (connec- 
tif) + nom, comme c'est le cas avec les noms composés Abeñy ndame, Abeñy $n t s a p$, etc. en l'exemple 4 . Cette question aurait dû être réglée dans les prétextes.

Son corollaire est le fait que jusqu'à ce jour, il n'y a aucun système d'orthographe uniformisé pour le fang et même à l'échelle de l'ensemble des « langues du Gabon ». La proposition faite en 1999 sous la désignation d'Orthographe des Langues Gabonaises (1999) n'a pas, elle non plus malheureusement, réglé le problème de l'écriture des mots composés, ainsi que celle des possessifs, démonstratifs, et nombre d'autres catégories grammaticales.

(4) Le traitement des homonymes souffre, lui aussi, d'insuffisances. Considérons à ce sujet l'exemple ci-après:

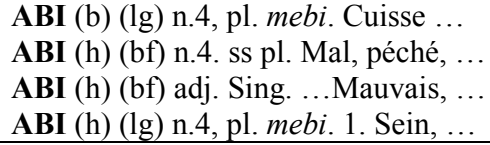

Exemple textuel 10: Article ABI (h), ABI (h) et ABI (h) (extrait du Galley 1964: 17)

Les trois dernières unités de traitement du paradigme mentionné ci-dessus, qui, selon le lexicographe, ont la même indication de tonalité (cf. (h)), ont reçu le statut de lemmes, comme homonymes. Le dictionnaire n'aide pas son utilisateur-cible à distinguer les trois membres du paradigme homonymique. L'auteur aurait pu les affecter de chiffres en exposant, ou alors, commencer par un indicateur structurel, reflétant la fréquence d'emploi comme cela est habituellement fait dans de tels cas. Par exemple, $\mathbf{A B I}^{1} ; \mathbf{A B I}^{2} ; \mathbf{A B I}^{\mathbf{3}}$ ou $\mathbf{1}$. ABI; 2. ABI; 3. ABI. De nombreux exemples pourraient être appelés pour illustrer cette lacune systématique dans le dictionnaire.

(5) Les explications que fournit la paraphrase de signification mettent certes en exergue quelques aspects culturels de la vie des Fang et permettent à l'utilisateur de tirer beaucoup d'informations utiles de ces entrées, mais il faut constater qu'elles surchargent le texte.

L'une des manières, pour les lexicographes, de rendre compte des explications culturelles en évitant que trop de données encyclopédiques s'infiltrent dans la paraphrase de la signification consiste à concevoir des articles de synthèse; en l'occurrence, des articles présentant une vue d'ensemble d'un champ de connaissance.

(6) L'article portant sur les termes de la faune et de la flore présente une structure peu commune dans le dictionnaire, et mériterait ainsi quelques explications dans l'un des textes externes du dictionnaire. 


\section{La structure d'accès du dictionnaire de Galley}

Comme dans le développement qui précède, nous traitons de la structure d'accès du dictionnaire de Galley en exposant les avantages et les inconvénients.

\subsection{Avantages}

On distingue généralement deux types de structures d'accès:

(a) structure d'accès externe; et

(b) structure d'accès interne.

La structure d'accès externe guide l'usager à partir d'un point de départ externe jusqu'au signe lemme. Par exemple, le titre du dictionnaire, la table des matières et les entêtes ressortissent à la structure d'accès externe. Il est bon de faire observer que la présentation des entêtes dans le dictionnaire de Galley a grandement amélioré sa structure d'accès externe.

La structure d'accès interne, d'autre part, commence à partir du signe lemme et se poursuit à travers l'article du dictionnaire jusqu'à l'information recherchée. Pour s'assurer que l'usager aura un accès rapide et facile aux données présentées, les lexicographes utilisent généralement des marqueurs structurels typographiques et non-typographiques. Pour ce qui est des indicateurs structurels typographiques, dans le dictionnaire de Galley, les lemmes apparaissent en gras et en " petites majuscules ». Les significations des lemmes ainsi que les traductions françaises des phrases en fang sont présentées en romain. Par contre, les items lexicaux, les syntagmes et les phrases en fang sont présentés en italique. L'utilisation de tous ces indicateurs structurels typographiques facilite aux usagers l'accès aux données recherchées.

\subsection{Inconvénients}

(1) Les marqueurs structurels non-typographiques incluent un certain nombre de symboles, dont, et entre autres: les diamants noirs, le triangle noir à l'envers, les rectangles, souvent utilisés pour marquer les limites d'une zone de recherche spécifique (définition, exemple, catégorie grammaticale ...). Le dictionnaire de Galley présente à ce sujet peu de marqueurs structurels non-typographiques, alors que ceux-ci facilitent généralement aux usagers l'accès aux données recherchées.

(2) Le texte souffre aussi au niveau du traitement des éléments de la littérature sentencieuse (cf. proverbes, maximes, adages, ...). Les proverbes par exemple sont traités dans la nomenclature, et notamment dans l'article du lemme introduisant ou figurant dans le proverbe traité. Mavoungou (2002: 230-237) nous montre au contraire que les proverbes et les autres expressions idiomatiques 
n'appartiennent pas, à proprement parler, à la nomenclature; pour lui, ils doivent être traités dans les textes externes du dictionnaire et de préférence dans les posttextes. Un tel traitement permet de créer une autre possibilité d'accès pour l'utilisateur, et donne au dictionnaire un caractère polyaccessible.

\section{Conclusion}

À un degré important, les pré-textes du dictionnaire fournissent assez d'informations concernant les sources primaires de l'ouvrage. Cependant, l'auteur ne donne pas une indication de ses sources secondaires. Il n'y a aucun doute que le lexicographe a employé un corpus lexicographique volumineux de la langue, ce qui fait du dictionnaire une source valable de référence et un véritable réservoir de connaissances. Les éléments macrostructuraux ont été sélectionnés parmi divers champs de connaissance (la tradition orale, la religion, la sylviculture, la pharmacopée traditionnelle, entre d'autres). Malheureusement, le lexicographe ne donne pas les critères qui ont présidé à l'incorporation des items lexicaux. En outre, la macrostructure ne présente pas de caractéristiques de sophistication, c'est-à-dire, l'utilisation des procédures de «niches » et de «nids ». La microstructure contient beaucoup d'entrées utiles, mais aucune discussion métalexicographique n'est fournie dans le guide aux usagers pour rendre l'ouvrage accessible au lecteur-cible.

\section{Notes}

1. Opuscule centenaire de 14 pages "premier livre de fang " dit le titre. C'est la mission protestante de Talagouga (près de Ndjolé) qui est à l'origine de cette publication dont la reliure est assurée par du fil à aiguille. Etrange illustration de couverture: minarets et chéchias composent un paysage sans doute exotique mais fantastiquement irréaliste (Mayer 1990: 74).

2. Autre désignation du Dictionnaire fang-français, français-fang de Samuel Galley.

3. Localité située dans les environs de Ndjolé.

4. Encore appelées corpus lexicographique ou base du dictionnaire, qui incluent toutes les sources qui ne sont pas des dictionnaires eux-même (cf. Smit 1996: 30-31 et 111).

5. Tous les dictionnaires consultés durant la phase de rédaction (cf. Wiegand et Kučera 1981: 100ff).

6. Marling (1872), Lejeune (1892) et Martrou (1924).

\section{Références bibliographiques}

Bureau du WAT. 1999. Study Guide of the Woordeboek van die Afrikaanse Taal (WAT). Notes de cours non-publiées. Stellenbosch: Bureau du WAT.

Galley, S. 1889. Nteni Osi Nteni Fañwe. Talagouga: Imprimerie de la Mission.

Galley, S. 1964. Dictionnaire fang-français et français-fang, suivi d'une grammaire fang. Neuchâtel: Henri Messeiller. 
Hausmann, F.J. et R.O. Werner. 1991. Spezifische Bauteile und Strukturen zweisprachiger Wörterbücher: eine Übersicht. Hausmann, F. J. et al. (Éds.). 1989-1991: 2729-2769. Berlin: Walter de Gruyter.

Hausmann, F.J. et H.E. Wiegand. 1989. Component Parts and Structures of General Monolingual Dictionaries: A Survey. Hausmann, F.J. et al. (Éds.). 1989-1991: 328-360. Berlin: Walter de Gruyter.

Hausmann, F.J., O. Reichmann, H.E. Wiegand et L. Zgusta (Éds.). 1989-1991. Wörterbücher. Ein internationales Handbüch zur Lexikographie/Dictionaries. An International Encyclopedia of Lexicography/Dictionnaires. Encyclopédie internationale de lexicographie. Handbücher zur Sprach- und Kommunikationswissenschaft 5.1-5.3. Berlin: Walter de Gruyter.

Largeau, V. 1901. Encyclopédie pahouine. Eléments de grammaire et dictionnaire français-pahouin. Paris: E. Leroux.

Lejeune, L. 1892. Dictionnaire français-fang ou pahouin, précédé de quelques principes grammaticaux sur cette même langue. Paris: A. Faivre et H. Teillard.

Marling. 1872. Dictionnaire fang-français. New York.

Martrou, L. 1924. Lexique fãn-français. Procure Générale (des Pères du St Esprit) Abbeville. Paris: Imprimerie Paillard.

Mavoungou, P.A. 2001. Macro- and Microstructural Issues in Mazuna Lexicography. Lexikos 11: 122-138.

Mavoungou, P.A. 2002. Metalexicographical Criteria for the Compilation of a Trilingual Dictionary: Yilumbu-English-French. Thèse de doctorat non-publiée. Stellenbosch: Université de Stellenbosch.

Mayer, R. 1990. Histoire de l'écriture des langues du Gabon. Revue Gabonaise des Sciences de l'Homme 2: 65-91.

McArthur, T. 1986. Worlds of Reference. Lexicography, Learning and Language from the Clay Tablets to the Computer. Cambridge: Cambridge University Press.

Mihindou, G.-R. 2001. Apports des missionnaires à la lexicographie gabonaise: dictionnaires bilingues fang-français/français-fang; français-yipounou/yipounou-français; français/mpongwé. Emejulu J.D. (Éd.). 2001. Éléments de lexicographie gabonaise. Tome I: 7-37. New York: JimacsHillman Publishers.

Smit, M. 1996. Wiegand's Metalexicography as a Framework for a Multilingual, Multicultural, Explanatory Music Dictionary for South Africa. Thèse de doctorat non-publiée. Stellenbosch: Université de Stellenbosch.

Wiegand, H.E. et A. Kučera. 1981. Brockhaus-Wahrig: Deutsches Wörterbuch auf dem Prüfstand der praktischen Lexikologie. I. Teil: 1. Band (A-BT); 2. Band (BU-FZ). Kopenhagener Beiträge zur germanistischen Linguistik 18: 94-217. 